\title{
Learning Network Topology from Simple Sensor Data
}

\author{
Dimitri Marinakis, Philippe Giguère, and Gregory Dudek \\ Centre for Intelligent Machines, McGill University, \\ 3480 University St, Montreal, Quebec, Canada H3A 2A7 \\ \{dmarinak, philg, dudek\}@cim.mcgill.ca \\ http://www.cim.mcgill.ca
}

\begin{abstract}
In this paper, we present an approach for recovering a topological map of the environment using only detection events from a deployed sensor network. Unlike other solutions to this problem, our technique operates on timestamp free observational data; i.e. no timing information is exploited by our algorithm except the ordering. We first give a theoretical analysis of this version of the problem, and then we show that by considering a sliding window over the observations, the problem can be re-formulated as a version of set-covering. We present two heuristics based on this set-covering formulation and evaluate them with numerical simulations. The experiments demonstrate that promising results can be obtained using a greedy algorithm.
\end{abstract}

Key words: sensor networks, topology inference, set-covering, mapping

\section{Introduction}

In this paper we consider the problem of learning the topology of a network of sensors through the exploitation of motion in the environment. We assume that an individual sensor is capable of detecting the passage of an agent through its local region, but is unable to generate a reliable signature. Our approach uses the combined observational data returned from our network to infer the topological relationships between the sensors.

We will illustrate the problem with a simplified example. Figure 1(a) depicts a sensor network distributed within an indoor environment. Let us assume that the network has been deployed for some purpose, such as surveillance, and requires knowledge of the inter-node connectivity in order to fulfill its function. During some initial calibration period the network collects observations of agents passing by each sensor (Figure 1(b)). The problem we are trying to solve is how to use these collected observations to construct the topological description of the network shown in Figure 1(c). This type of network might arise if wireless cameras were deployed in a workplace environment.

In this work, we assume not only that the agents moving though the environment are indistinguishable, but that there are no temporal clues that can be used to aid the inference process. In other words, the detection events are correctly ordered but are not time-stamped. Therefore, when our inference algorithm is employed, the time-stamp data can be discarded or simply not collected in the first place. In order to exploit timing information in the observational sequence some model of agent motion in the environment needs to be either constructed based on prior assumptions, or learned from the 


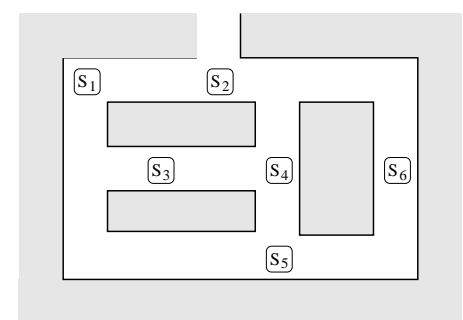

(a)

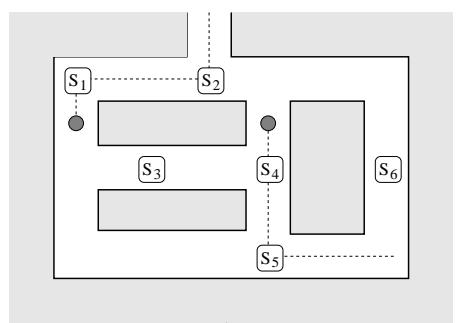

(b)

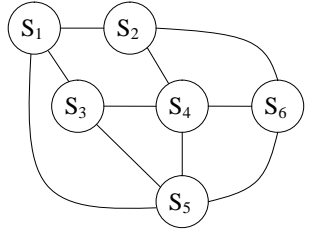

(c)

Fig. 1. An example of a sensor network which we wish to calibrate. a) The original ad-hoc deployment. b) An Example of agent motion exploited by the calibration process. c) The desired topological connectivity map of the network.

data. Our technique, however, allows the correct edges in the graph to be inferred while avoiding the prior domain knowledge or algorithmic complications involved in constructing an adequately accurate motion model. By employing a sliding window over the observations, we will show that the problem can be re-formulated as a version of the well understood set-covering problem and accurate results can be obtained without timing information.

The ability of a surveillance or monitoring system to automatically determine the connectivity parameters describing its environment is useful for a number of reasons. Although the topology information can be manually entered during installation, more detailed parameters such as the relative connectivity strength between links are difficult to determine, and a change in the environment or network would require re-calibration. Once calibrated, the connectivity information could aid in conventional target tracking and additional monitoring activities. For example, by reconstructing trajectories, a vehicle monitoring network distributed about a city could help make decisions about road improvements which might best alleviate congestion. In addition, the topological information could be combined with relative localization techniques [1] [2] [3] [4] to recover a more complete representation of the environment.

\section{Background}

Although the topological mapping problem has been well explored in mobile robotics [5] [6] [7] [8], most sensor network related investigations have been more recent [9] [10] [11] [12]. The outcome is generally a graph where vertices represent embedded sensors in the region and edges indicate navigability.

Ellis, Markis, and Black [9] approached the topology inference problem in the context of camera-based sensing. Their technique exploits temporal correlations in observations of agents' movements. They outlined an approach in which they first identified entrance and exit points in camera fields of view to generate a graph from video data. They then used a thresholding technique to look for peaks in the temporal distribution of travel times between entrance-exit pairs; a clear peak suggesting that the cameras 
are linked. This approach requires a large number of observations, but does not rely on object correlation across specific cameras. Thus, the method can be used to efficiently produce an approximate network connectivity graph.

Marinakis and Dudek [11] [12] have recently presented a solution to the topological inference problem that is based on a stochastic version of the Expectation Maximization algorithm. Their approach uses only detection events from the deployed sensors and is based on reconstructing plausible agents trajectories. Results presented from simulations and experimental data suggest that their technique produces accurate results under a variety of conditions and compares well to other approaches.

When the observation are information-poor, topology inference through trajectory reconstruction has much in common with the data association problem in multi-object tracking and similar statistical techniques are employed. For example, in [13], event detections alone were used for the tracking of multiple targets using Markov Chain Monte Carlo (MCMC). Similarly, [14] approached a traffic monitoring problem using limited sensor data observations through a stochastic sampling approach.

A key observation regarding all of the approaches mentioned above is that their performance will suffer if temporal information is removed from the observations. Ellis, Markis, and Black rely explicitly on this temporal data, while the approaches employing probabilistic frameworks [11] [13] [14] exploit the delay information to aid in the data association problem.

In the remainder of this paper, we consider the problem of solving the topology inference problem, relying only on the ordering of the timing information in the observational data. We discuss theoretical aspects of this version of the problem and present an algorithm for its solution. It is our hope that concepts presented here can be incorporated into more general techniques for topology inference, or used in their own right.

\section{Problem Definition}

We formulate the problem of learning the network topology as the inference of a directed graph $G=(V, E)$, where the vertices $V=v_{i}$ represent the locations where sensors are deployed, and the edges $E=e_{i, j}$ represent the connectivity between them; an edge $e_{i, j}$ denotes a path from the position of sensor $v_{i}$ to the position of sensor $v_{j}$. The sources of motion in the sensor network are modeled as some number $N$ of agents moving asynchronously through the graph. Each agent generates an observation every time it visits a vertex. This corresponds to an agent passing near a particular sensor which then detects the presence of motion in its region.

The input to the problem is an ordered list of observations $O=\left\{o_{t}\right\}$, each of which is identifiably generated by one of the sensors; i.e. each $o_{t} \in[1, V]$. The goal is to find the correct underlying graph $G$ explaining this observational sequence. 


\section{Algorithm Formulation}

\subsection{Smallest Graph is Correct Answer}

The key idea behind our approach is to find the smallest ${ }^{1}$ graph that successfully explains the observed data. Leaving aside for the moment the actual implementation details, let us consider this idea in more depth by proposing the existence of an algorithm $A$ that takes as an input the assumed number of agents $N^{\prime}$ in the environment and the observational sequence and returns as an output the smallest graph consistent with the observations.

Our algorithm $A$ considers each of the possible trajectories that could be taken by these $N^{\prime}$ agents given the observational sequence and then selects the trajectory set that requires the smallest number of inter-vertex traversals. The algorithm then returns the graph populated only with edges that correspond to the inter-vertex traversals required by this chosen trajectory set.

The concept that the simplest solution explaining the data is probably the correct solution has been used successfully in a different version of the topology inference problem [12]. The principle, known as Occam's razor, states, "if presented with a choice between indifferent alternatives, then one ought to select the simplest one." The concept is a common theme in computer science and underlies a number of approaches in AI; e.g. hypothesis selection in decision trees and Bayesian classifiers [15]. We will show in the next section that under certain assumptions, we can prove that an algorithm trying to find the smallest graph will return the correct answer.

\subsection{Correctness of the Smallest Graph Assumption}

In this section we present a proof that the smallest graph $G$ consistent with the observations is the correct solution $G_{c}$ given the following assumptions:

1. There are an infinite number of observations, $O$.

2. The motion of each of the agents is random.

3. The true number of agents in the system $N$ is fixed and bounded by the number assumed by the algorithm $A$; i.e. $N<=N^{\prime}$.

4. The transit time between nodes is un-bounded.

5. There are no self-referential connections in the true graph $G_{c}$; i.e. no agent may trigger two observations by one passage through the region of a single sensor.

\subsection{Proof of Smallest Graph}

First, we will prove that there exist no graph smaller than $G_{c}$ that can explain the observed data, given the above stated assumptions. This is done by showing that it is possible to have sequences generated by $G_{c}$ that cannot be explained by this smaller graph $G_{c}^{\prime}$. In other words, $G_{c}^{\prime}$ is not consistent with the observations, and by definition cannot be a solution.

\footnotetext{
${ }^{1}$ The graph with the smallest number of edges.
} 


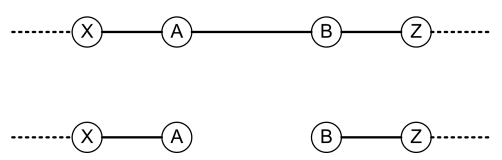

Fig. 2. Example of removing edge $\mathrm{AB}$ from graph $G_{c}$, (shown partially on top), to create graph $G_{c}^{\prime}$, (shown partially below).

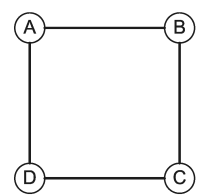

a)

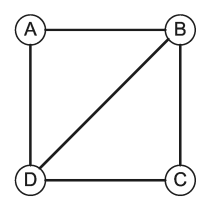

Fig. 3. a) The correct graph $G_{c}$ b) an incorrect graph

Let us consider a graph $G_{c}^{\prime}$ created by removing a single edge from $G_{c}$, as in figure 2. In this case, we remove the edge $A B$ from graph $G_{c}$. Let us now create a valid observational sub-sequence $O=A B A B A B A B \ldots A B$ which was created in truth by a single agent traversing back and forth on the edge $A B$. The only way agents in a graph $G_{c}^{\prime}$ could generate this observational sequence would be if some number of them were 'stationed' at node $X$, and some number 'stationed' at node $Z$, and alternatively one agent from $X$ traversed the edge to $A$, and then one from $Z$ traversed the edge to $B$. However, if the length, $|O|$ of the observational sub-sequence $O$ is larger than the maximum possible number of agents $N^{\prime}$, then there will not be enough agents in $G_{c}^{\prime}$ to generate $O$. Therefore, the edge $A B$ must be present in any consistent solution. Applying this to all the edges in $G_{c}$, we see that a solution that can explain all the transitions must have at least all the edges in $G_{c}$. Consequently, the smallest consistent graph is the correct graph $G_{c}$.

Note that this analysis requires that there be both an infinite number of observations and random motion on the part of the agents in order to allow such very rare observational sequences to exist. However, this concept holds with less formality to very large sequences of observations. It becomes less likely for a graph to successfully explain an observation sequence while missing portions of the real graph as the number of observations increases.

\subsection{Impact of Estimated Number $N \leq N^{\prime}$ of Agent on Solution $G$}

In the following sections, we will show the impact of the number $N$ of agent used to find a solution. More precisely, we will show that if an algorithm overestimates the number $N$ of agents in the system, it will still give the correct answer $G_{c}$, but not if it underestimates it.

Lemma 1 Overestimating the number $N$ of agents while looking for the smallest graph results in the correct solution. 
To prove this lemma, we will show that a path generated by a single agent can be spliced between two agents using a 'tag team' method, and yet will still a yield a the correct graph $G_{c}$. That way, all superflouous agents used in the algorithm can be 'hidden' by splicing a valid path repeatedly.

Let us consider a true sequence of vertex traversals $S$ generated by a single agent. First, without loss of generality, select any vertex $v_{\text {splice }} \in S$ as a splicing node. We can now pair any two virtual agents together to generate this traversal sequence $S$ in the following way. Let one of the virtual agent be initially stationed at $v_{\text {splice }}$. When the other virtual agent enters this vertex it will exchange its role with the first agent, as in a game of tag team wrestling. The other agent will now leave the vertex $v_{\text {splice }}$, generating a sub-sequence of $S$ until it re-enters $v_{\text {splice }}$, where again they will switch roles.

As an example, let us consider the vertex sequence $S=A B C D A D C D A B C B A$ generated by a true agent in $G_{c}$ of Figure 3(a.). We choose $v_{\text {splice }}=C$. Now the vertex sequence $S$ assumed to come from a single agent looks like the following: $A B$ cdad $C D A B$ cba where capital letters are used for the path $P_{1}$ of agent one, and small bold letters are used for the path $P_{2}$ of agent two. The individual virtual sequences $P_{1}=A B C D A B$ and $P_{2}=$ cdadcba are both valid sequences in the correct graph $G_{c}$.

This analysis shows that we can assume the existence of more agents than the number that actually generated the observation sequence, and still produce paths that are consistent with the correct graph.

Lemma 2 Underestimating the number of agents can result in an incorrect solution.

To prove this lemma, we will simply show that there exists at least one observational sequence $O$ such that underestimating the number of agents creates a false solution. Let us consider again the graph depicted in figure 3(a.) and let us consider the motion of two agents in this graph. Agent one will follow the path $A B C D A D C$, and agent two will follow the path $\mathbf{d b}$. By combining the two paths, it is possible to get the sequence of observations: $O_{1}=A B \mathbf{d} C D A \mathbf{b} D C$. If we assumed the existence of only one agent, then the smallest graph that can explain the transitions $O_{1}$ is displayed in figure 3(b.) and is incorrect.

Using the above stated two lemma, we arrive at the following theorem (which holds true given the earlier stated assumptions):

Theorem 1 If the number of assumed agents $N^{\prime}$ in the algorithm is equal or greater than the true number of agents $N$ that generated the observations, then the smallest graph consistent with the observations will be the correct graph $G_{c}$. If the number of assumed agent is smaller than the true number, then there are no guarantees that the smallest graph consistent with the observations will be the correct graph $G_{c}$.

In the next section, we draw on this theoretical analysis to motivate a pragmatic approach for topology inference. 


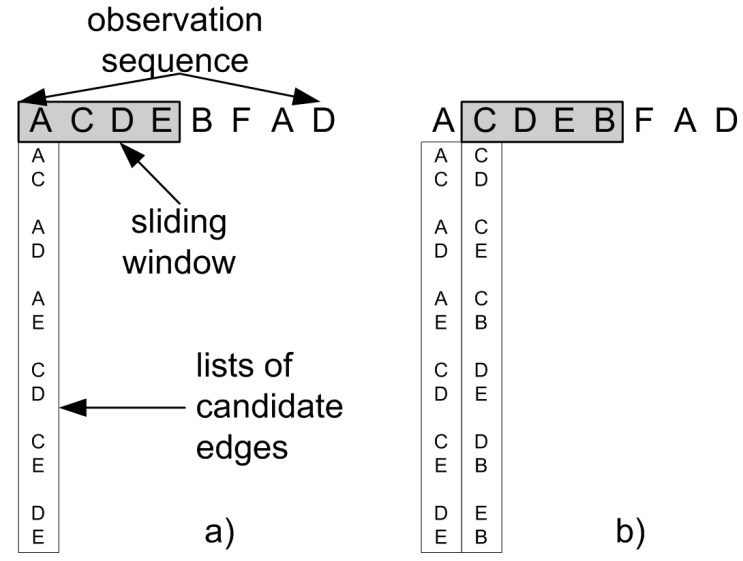

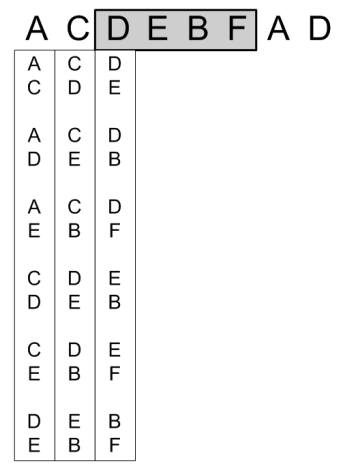

c)

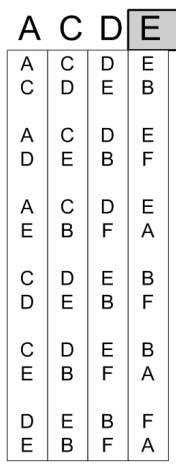

d)

Fig. 4. Example of generating candidate edges for each sliding window position. The window is moved to the right from a) to d).

\section{The Sliding Window Approach}

We now present an algorithm for estimating the smallest possible graph given an observation sequence. Our approach is based on the following lemma:

Lemma 3 In any given continuous sequence of $N_{S}>N$ observations, at least $\left(N_{S}-\right.$ $N)$ transitions between observations correspond to edges in the correct graph $G_{c}$.

For example, let $N_{S}=4$ and $N=3$ and the recorded observational sequence be $A B C D$. The possible transitions between nodes are $A B, A C, A D, B C, B D$ and $C D$. Since we have one more observation than the number of agents, $\left(N_{S}-N=1\right)$, it means that at least one agent must have generated more than one of the observations in this sequence, and therefore at least one of the transitions listed above must be valid; i.e. 
present in $G_{c}$. Note that the number of potential transitions generated with a sequence of $N_{S}$ observations is:

$$
N_{T}=\frac{\left(N_{S}-1\right) N_{S}}{2}
$$

Our technique is to employ a sliding window of size $N_{S}=N+1$ to consider in turn small continuous subsequences of the entire observation sequence $O$. Each of these subsequences gives rise to a list of $N_{T}=\left(N^{2}+N\right) / 2$ candidate edges $L_{i}$, one of which must be present in the true solution $G_{c}$. Once the window has moved over the complete observation sequence $O$, there will be $K=|O|-N_{S}$ lists generated. Figure 4 shows an example of generating candidate edges using the sliding window approach. Motivated by Theorem 1, our approach is find the smallest graph that can explain at least one edge in each in each of these candidate lists: $L_{1}, L_{2}, \ldots L_{K}$.

This problem can be shown to be equivalent to the set-covering problem which is NP-complete, however, several heuristics can be employed to estimate an optimal solution. We will consider a two heuristic approaches in the next sections.

\subsection{A Greedy Approach}

One method of obtaining a solution to the sliding window problem posed above, is to adopt a greedy approach. This is a standard heuristic often used with good results for set-covering problems. In our domain, the greedy algorithm would work as follows:

1. Begin by marking all candidate lists $L_{1}, L_{2}, \ldots L_{K}$ unexplained and initialize a list of edges $E$ to be empty.

2. Find the edge $e$ that is present in the greatest number of currently unexplained candidate lists.

3. Remove from consideration those candidate lists which contain edge $e$ by marking them explained, and add $e$ to $E$.

4. Repeat steps 2 to 3 until all lists are marked explained. Return the graph corresponding to our list of edges $E$ as the final solution.

\subsection{A Statistical Approach}

A statistical approach could also be used to determine the correct edges in $G_{c}$. The number of times a given edge has been seen in any candidate list could be tallied up. Those edges that occur with a frequency greater than some threshold $T$ could then be selected.

Let us consider a suitable value for the threshold $T$. If $G_{c}$ corresponded to a fully connected undirected graph, the average tally of each edge would be:

$$
\mu=\frac{K N_{T}}{|E|}
$$

where $N_{T}$ is the number of candidate edges generated per window, $K$ is the number of candidate lists (windows), and $|E|$ is the number of potential edges in the graph. Replacing $K$ with $|O|-N_{S}, N_{T}$ with $\left(N^{2}+N\right) / 2$, and $|E|$ with $V(V-1)$, we arrive at: 


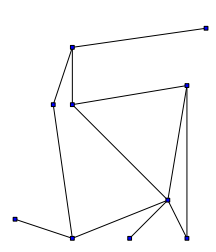

(a)

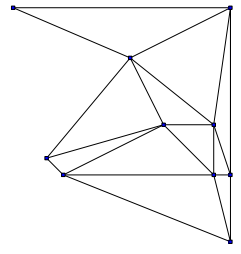

(b)

Fig. 5. Example of graphs created using the Delaunay triangulation technique: a) 10 node graph with 12 edges, b) 10 node graph with 20 edges

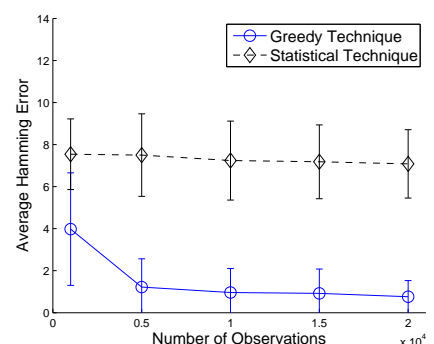

(a)

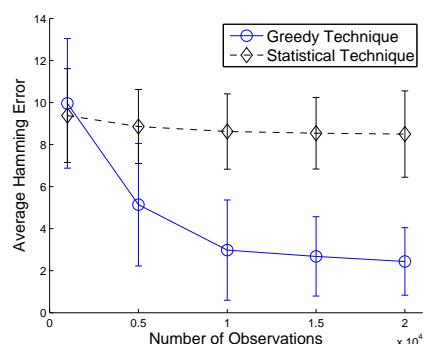

(b)

Fig. 6. Mean Hamming Error obtained from the two techniques for various numbers of observations averaged averaged over 50 randomly produced graphs. (Error bars show one standard deviation). Results obtained from 10 node graphs with: a) 12 edges b) 20 edges

$$
\mu=\frac{\left(|O|-N_{S}\right)\left(N_{S}-1\right) N_{S}}{V(V-1)}
$$

Since we expect $G_{c}$ to contain less edges than its fully connected counterpart, $T=$ $\mu$ can be expected to be a suitable threshold.

\section{Experiments}

\subsection{Simulator}

We have examined the sliding window approach with a number of experiments conducted in simulation. We have constructed a simulation tool that takes as input a graph and the number of agents in the environment and outputs a list of observations generated by randomly walking the agents through the environment. A number of experiments were run using this simulator on randomly generated planar, connected graphs (Figure 


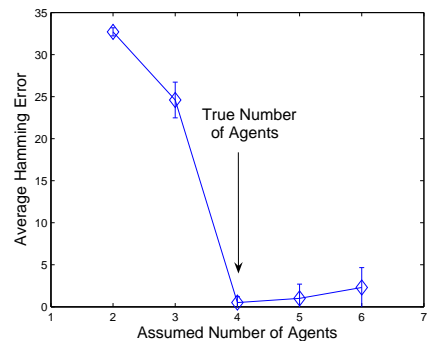

(a)

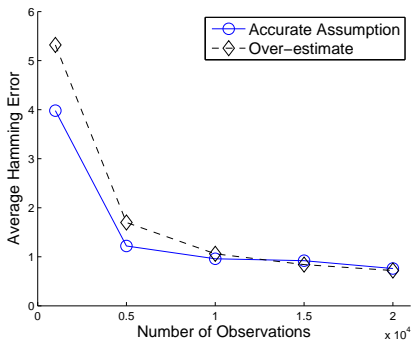

(b)

Fig. 7. Results obtained by differing the assumed number of agents for graphs of size 10 nodes and 14 edges. a.) Hamming Error as a function of the assumed number of agents for the greedy algorithm. Results obtained with 10000 observations generated from 4 agents and averaged over 10 graphs. (Error bars show one standard deviation). b.) Mean Hamming Error as a function of observations for an accurate assumption of 4 agents and an over-estimate of 5 agents. Results averaged over 50 graphs.

5). The graphs were produced by selecting a connected sub-graph of the Delaunay triangulation of a set of randomly distributed points. For each experiment, the results were obtained by considering the Hamming error between the true and inferred graph.

\subsection{Results}

The greedy approach was capable of producing accurate results in moderately sized graphs with a reasonable number of agents, given an adequate number of observations. Although not as accurate on average as the greedy approach, the statistical approach was also capable of producing a solution near the true answer. Figure 6 compares the accuracy of theses two approaches over 50 randomly produced graphs of 10 nodes and two different edge densities. Note that the accuracy of both approaches tended to increase as the number of observations increased. It also appeared that denser graphs required larger numbers of observations to obtain the same accuracy level than that obtained in sparser graphs. Additionally, it was observed that the greedy approach obtained a better Hamming error on average for less dense graphs. However, when the proportion of the true graph structure recovered was considered, this effect was lessened. For example, for the experiment shown in figure 6, the Hamming error divided by the true number of edges in the graph was approximately double for denser graph, while the Hamming error alone was approximately triple.

Unsurprisingly, the accuracy of the statistical approach was very sensitive to the value of the threshold selected. Experiments not shown here verified that the value for $T$ selected above was generally suitable for graphs of various densities and sizes, although often better results could be obtained for any specific graph type through careful tuning. This approach requires relatively little computational effort and might have value as a bootstrapping technique for more complex approaches such as the one presented in [11]. 


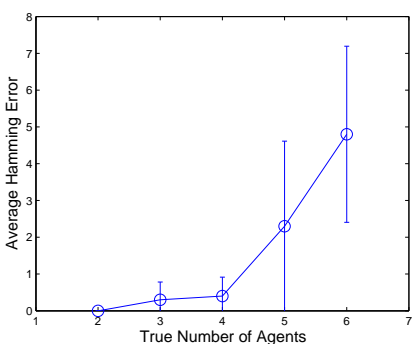

(a)

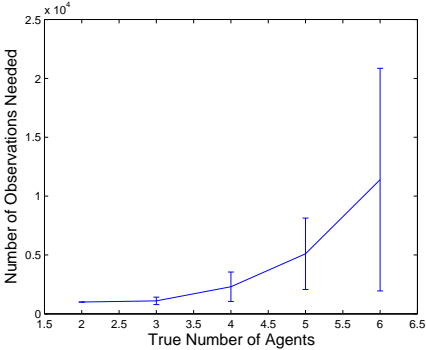

(b)

Fig. 8. Performance of algorithm as a function of the true number of agents for the greedy algorithm where the assumed number of agents is set to the correct number. Results averaged over 10 graphs of size 10 nodes and 14 edges; (error bars show one standard deviation). a.) Hamming Error obtained with 10000 observations. b.) Number of observations required to obtain a result with a Hamming error of 2 or less.

As predicted by theorem 1, the effect of over-estimating the number of agents in the environment was indeed less detrimental than that of under-estimating the number of agents. Figure 7 shows the result of assuming various numbers of agents in one situation for the greedy approach. As the over-estimation increases, the required number of observations needed to solve the problem also increases.

The problem of topology inference becomes more difficult as more agents are added to the system. Figure 8 shows the correspondingly poorer performance obtained with the greedy approach on the same set of graphs with observations generated from different numbers of agents. Even if the correct number of agents is known, we suspect that less information is available as the number of agents increases. As the size of the sliding window increases, so does the number of candidate edges generated by each sliding window. Therefore, the ratio of known correct to incorrect edges decreases, and hence, more observations are needed to obtain the same level of error.

\section{Conclusion}

In this paper, we have described a way of learning the topology of a sensor network, using only event ordering information. We presented a theoretical analysis of the problem, and re-formulated it as a set-covering problem. Two methods were presented to solve this problem, one based on statistics, and one based on a sliding window technique. We explored the effectiveness of both approaches through numerical simulations for various test cases. Our work demonstrates the promise of this approach for topology inference.

In future work, we hope to extend some of our theoretical results. We would be interested in furthering our understanding regarding the impact of the number of agents in the system since this value tends to dilute the information gained per each observation. This effort would entail deriving a relationship for the information gained in the 
context of the sliding window approach. Additionally, it would be of interest to find an analytical relationship between the number of observations needed to solve a problem and the corresponding density of agents in the system; i.e. the ratio of agents to edges in the true graph. On a different level, we would like to apply the findings presented in this paper to the version of the problem where timing data is available and compare it to other established methods.

Acknowledgments. We would like to acknowledge Ketan Dalal for his helpful analysis of this problem.

\section{References}

1. Savvides, A., Han, C., Strivastava, M.: Dynamic fine-grained localization in ad-hoc networks of sensors. In: 7th annual international conference on Mobile computing and networking, Rome, Italy (2001) 166-179

2. Moore, D., Leonard, J., Rus, D., Teller, S.: Robust distributed network localization with noisy range measurements. In: Proc. of the Second ACM Conference on Embedded Networked Sensor Systems (SenSys '04), Baltimore (2004)

3. Marinakis, D., Dudek, G.: Probabilistic self-localization for sensor networks. In: AAAI National Conference on Artificial Intelligence, Boston, Massachusetts (2006)

4. Ihler, A.T., Fisher III, J.W., Moses, R.L., Willsky, A.S.: Nonparametric belief propagation for self-calibration in sensor networks. IEEE Journal of Selected Areas in Communication (2005)

5. Shatkay, H., Kaelbling, L.P.: Learning topological maps with weak local odometric information. In: IJCAI97, San Mateo, CA (1997) 920-929

6. Choset, H., Nagatani, K.: Topological simultaneous localization and mapping (SLAM): toward exact localization without explicit localization. IEEE Transactions on Robotics and Automation 17(2) (2001) 125 - 137

7. Remolina, E., Kuipers, B.: Towards a general theory of topological maps. Artif. Intell. 152(1) (2004) 47-104

8. Ranganathan, A., Dellaert, F.: Data driven MCMC for appearance-based topological mapping. In: Proceedings of Robotics: Science and Systems, Cambridge, USA (2005)

9. Makris, D., Ellis, T., Black, J.: Bridging the gaps between cameras. In: IEEE Conference on Computer Vision and Pattern Recognition CVPR 2004, Washington DC (2004)

10. Rekleitis, I., Meger, D., Dudek, G.: Simultaneous planning localization, and mapping in a camera sensor network. Robotics and Autonomous Systems (RAS) Journal, special issue on Planning and Uncertainty in Robotics (2006)

11. Marinakis, D., Dudek, G.: A practical algorithm for network topology inference. In: IEEE Intl. Conf. on Robotics and Automation, Orlando, Florida (2006)

12. Marinakis, D., Dudek, G.: Topological mapping through distributed, passive sensors. In: International Joint Conference on Artificial Intelligence, Hyderabad, India (2007)

13. Songhwai Oh, Phoebus Chen, M.M., Sastry, S.: Instrumenting wireless sensor networks for real-time surveillance. In: Proc. of the International Conference on Robotics and Automation. (2006)

14. Pasula, H., Russell, S., Ostland, M., Ritov, Y.: Tracking many objects with many sensors. In: IJCAI-99, Stockholm (1999)

15. Michell, T.M.: Machine Learning. McGraw-Hill, Boston (1997) 